\title{
BMJ Open Prospective economic evaluation of an electronic discharge communication tool: analysis of a randomised controlled trial
}

\author{
Laura K Sevick, ${ }^{1}$ Maria-Jose Santana, ${ }^{1,2,3}$ William A Ghali, ${ }^{1,2,3,4}$ Fiona Clement ${ }^{1,2}$
}

To cite: Sevick LK, Santana M-J, Ghali WA, et al. Prospective economic evaluation of an electronic discharge communication tool: analysis of a randomised controlled trial. BMJ Open 2017;7:e019139. doi:10.1136/ bmjopen-2017-019139

- Prepublication history for this paper is available online. To view these files, please visit the journal online (http://dx.doi. org/10.1136/bmjopen-2017019139).

Received 24 August 2017 Revised 26 0ctober 2017 Accepted 14 November 2017

\section{CrossMark}

${ }^{1}$ Department of Community Health Sciences, University of Calgary, Calgary, Alberta, Canada

${ }^{2} 0$ 'Brien Institute for Public Health, University of Calgary, Calgary, Alberta, Canada ${ }^{3}$ Medical Ward of the 21st Century, University of Calgary, Calgary, Alberta, Canada ${ }^{4}$ Department of Medicine, University of Calgary, Calgary, Alberta, Canada

Correspondence to

Dr Fiona Clement:

fclement@ucalgary.ca

\section{ABSTRACT}

Objective To complete an economic evaluation within a randomised controlled trial (RCT) comparing the use of an electronic discharge communication tool (eDCT) compared with usual care.

Setting Patients being discharged from a single tertiary care centre's internal medicine Medical Teaching Units.

Participants Between January 2012 and December 2013, 1399 patients were randomised to a discharge mechanism. Forty-five patients were excluded from the economic evaluation as they did not have data for the index hospitalisation cost; 1354 patients contributed to the economic evaluation.

Intervention eDCT generated at discharge containing structured content on reason for admission, details of the hospital stay, treatments received and follow-up care required. The control group was discharged via traditional dictation methods.

Primary and secondary outcome measures The primary economic outcome was the cost per qualityadjusted life year (QALY) gained. Secondary outcomes included the cost per death avoided and the cost per readmission avoided.

Results The average transcription cost was \$C22.28 per patient, whereas the estimated cost of the eDCT was \$C13.33 per patient. The cost per QALY gained was \$C239 933 in the eDCT arm compared with usual care due to the very small gains in effectiveness and approximately $\$ C 800$ difference in resource utilisation costs. The bootstrap analyses resulted in eDCT being more effective and more costly in $29.2 \%$ of samples, less costly and more effective in $29.2 \%$ of samples, less effective and more costly in $23.9 \%$ of samples and finally, less costly and less effective in $17.7 \%$ of samples.

Conclusions The eDCT reduced per patient costs of the generation of discharge summaries. The bootstrap estimates demonstrate considerable uncertainty supporting the finding of neutrality reported in the clinical component of the RCT. The immediate transcription cost savings and previously documented provider and patient satisfaction may increase the impetus for organisations to invest in such systems, provided they have a foundation of eHealth infrastructure and readiness.

Trial registration number NCT01402609.

\section{Strengths and limitations of this study}

- This work reports the final prespecified outcome of a randomised controlled trial (RCT) to complete reporting of all trial outcomes.

- The assessment of economic value completes the consideration of the policy question of 'should organizations invest in an e-DCT?' through the lens of the quadruple aim.

- This economic evaluation follows best practice guidelines for economic evaluations.

- Costs of electronic discharge communication tool (eDCT) may not be generalisable. Estimates for the cost of discharge mechanism are context specific and will depend on the number of patients discharged, the existing infrastructure and the eDCT itself.

- The RCT permitted physician cross-over between trial arms. The true benefit of eDCT may have been underestimated, which would lead to an overestimation of the cost per quality-adjusted life year.

\section{BACKGROUND}

Recently, a multidimensional evaluation of an electronic discharge communication tool (eDCT) was completed. ${ }^{1}$ The study was designed as a two-arm randomised controlled trial (RCT). ${ }^{1}$ Aligned with the quadruple aim, ${ }^{2}$ there were four aspects of the evaluation within the RCT: (1) efficacy based on a composite primary outcome of death and readmissions, (2) provider satisfaction measured using the Questionnaire for User Interaction Satisfaction, ${ }^{3}$ (3) patient satisfaction using a brief survey and (4) economic value. ${ }^{4}$ The primary analysis demonstrated no difference in the primary outcome of death or readmissions between the eDCT and usual care arm resulting in neutral findings. ${ }^{1}$ However, there were positive findings for both patient and physician satisfaction. ${ }^{5}$ Thus, the policy question of 'should 
organizations invest in an e-DCT?' remains only partially answered without the final assessment of economic value.

An economic evaluation was designed a priori, following best practices, and was included in the original RCT protocol for the study described above. ${ }^{4}$ A recent systematic review assessing the economic literature found that there is limited evidence on the cost-effectiveness of eDCTs, and that none of the previous economic evaluations reported patient safety or quality of life outcomes. ${ }^{6}$ Thus, the primary objective of this paper was to assess the cost per quality-adjusted life year (QALY) gained comparing eDCTs to usual care for patients transitioning from acute care to community care. The reporting of the economic evaluation completes our reporting of all trial outcomes.

\section{METHODS}

\section{Overview of approach}

In keeping with best practices and the study protocol, a prospective economic evaluation of eDCT (treatment) compared with usual care (control) was conducted as part of the RCT. The RCT was a two-arm trial comparing eDCTs to usual care, defined as traditional discharge communication generated by dictation, for patients being discharged from a Canadian tertiary care centre's internal medicine medical teaching units (MTUs). ${ }^{1}$ These units provide care to non-surgical adult inpatients. Details of the full RCT methodology and results have been reported elsewhere. ${ }^{14}$ Between January 2012 and December 2013, 1953 patients were approached and screened for inclusion, and 1399 were randomised to discharge mechanism. ${ }^{1}$ Among these, 45 patients did not have data on cost for the index hospitalisation cost, so they were excluded from cost analyses. As a result, a total of 1354 patients contributed to the economic evaluation.

The RCT was powered to detect a $25 \%$ relative reduction in a composite of all-cause mortality or all-cause readmission within 90 days. ${ }^{1}$ The primary economic outcome was the cost per QALY with secondary outcomes of the cost per readmission gained and the cost per life saved. This is a deviation from the original study protocol; the cost per QALY is a more appropriate primary outcome to support allocative efficiency decisions. A healthcare public payer perspective was adopted over a 90-day time horizon. A 90-day time horizon was felt to be appropriate as it reflects a timeline where events could relate to discharge communication and is long enough to permit an event to occur. ${ }^{4}$ This is also a commonly reported time frame in readmission studies. ${ }^{4}$ All costs were inflated to 2016 Canadian dollars using the Canadian consumer price index.

\section{Cost of usual care}

Costs for traditional discharge generated by dictation was provided by estimates from the University of Calgary Medical Group Transcription Services. ${ }^{7}$ The base case costs assumed in-hospital dictation transcriptions are outsourced to an external provider (a common practice in many large healthcare systems). When outsourced, the total cost is calculated using the average cost per minute of dictation excluding technology fees. Ten physicians were asked to estimate the average length of a dictation. The average time was then used to calculate the total cost. The costs of a printer, printer toner and paper were excluded, as they would be negligible. Dissemination costs, including fax machines, were also excluded as these resources are commonly used in the physician offices for a variety of purposes and not exclusively for dictation discharges. All assumptions were explored in sensitivity analysis.

\section{Costs of eDCT}

To estimate the per person costs of eDCT, the disaggregated costs from the development, implementation and pilot testing of the previously described eDCT were considered. Reliability of the project estimate was confirmed by reviewing invoices and receipts. The total estimated cost was amortised over 3 years, which is reflective of the technology life-cycle. ${ }^{8}$ Monthly payments were calculated using an assumed $1 \%$ annual interest rate. ${ }^{9}$ The monthly payment amount was divided by the average number of discharges from the ward per day ( $\sim 9$ discharges/day) multiplied by 30 days. ${ }^{5}$ This daily average included all patients on the ward and not only study participants.

\section{Death, readmission and health-related quality of life}

Death and readmissions were modelled separately as these outcomes are valued differently and incur different resource utilisation. Death within 90 days postdischarge was captured through linkage to data from the Alberta Bureau of Vital Statistics. ${ }^{1}$ Readmissions within 90 days postdischarge were captured through data linkage to the AHS Data Integration, Measurement and Reporting unit. ${ }^{1}$ For both death and readmission, all-cause events were included, and avoidable and unavoidable events were not separated.

Health-related quality of life was measured at baseline, 30 days postdischarge, and 90 days postdischarge using the Health Utilities Index Mark 3 (HUI-3). Utility scores were calculated from the HUI-3 data using the HUI-3 Multiattribute Utility Function on the Dead-Healthy scale. ${ }^{10-12}$ To calculate QALYs at 90 days, the difference in utility score from baseline to 90 days was multiplied by 0.25 (reflective of the time horizon over 1 year of life).

\section{Healthcare resource utilisation and cost}

Index hospitalisation costs were estimated using microcosting data, which provide a detailed cost per patient including all resources consumed within the hospital stay such as nursing time, physician fees, drug costs and overhead costs. To estimate physician visits, physician claims and billing codes were used. Both the cost and number of readmissions were calculated. Patients who were readmitted to the same centre within 12 hours of discharge or another centre within 24 hours of discharge were 
assumed to be hospital transfers, not readmissions. Costs of the readmission hospitalisation were determined using gross costing methodology applied in the administrative data (Canadian Institute for Health Information Case Mix Grouper Plus). Ambulatory care utilisation and costs were determined by linking trial patients to the National Ambulatory Care Reporting System. Gross costing methodology was also applied (Canadian Institute for Health Information Comprehensive Ambulatory Classification System) ${ }^{13}$ Drug costs were estimated using data from the Pharmaceutical Information Network.

\section{Calculating the ICER}

Due to the differing numbers of patients included in each treatment arm $(n=679$ and $n=675)$, an average cost per patient was calculated. The average cost per patient includes the discharge mechanism (eDCT or usual care), the index hospitalisation, the readmissions, the emergency department (ED) visits, the ambulatory care visits, the drugs and the physician claims. The difference in the average cost per patient was then divided by the difference in the average clinical effect in each arm resulting in the incremental cost-effectiveness ratio (ICER). ${ }^{14}$

\section{Sensitivity and scenario analyses}

To examine the impact of uncertainty on the ICER, sensitivity and scenario analyses were conducted. Sensitivity analysis varied the costs of the eDCT $\pm 25 \%$. For the usual care arm, speech recognition systems or computer-aided medical transcription, that format draft documents to allow medical transcriptionists to edit and review rather than transcribe entire dictations may be used. Thus, a scenario analysis considering the costs of traditional discharge using internal (as opposed to outsourced) transcription with both a non-speech recognition system and a speech recognition system was completed. These analyses included the monthly technology fee, monthly outsourcing costs and monthly salary and benefits of transcriptionists (including overtime).

\section{Uncertainty bootstrapping analysis}

To examine the overall uncertainty, a bootstrapping analysis was completed. Moreover, to examine the variability in the total cost per patient and QALYs gained in each of the treatment arms, random samples of 50 patients for each of the trial arms were replicated 1000 times. The total cost per patient (including the cost of eDCT or usual care and all healthcare resource utilisation) as well as the average QALYs gained (between baseline and 90 days) were calculated for each sample. The cost and QALY difference between the two trial arms for each sample are plotted in a scatterplot to present the variability.

\section{RESULTS}

\section{Costs of usual care}

In the base case, outsourcing transcription costs to an external provider, the average cost per patient was
Table 1 Disaggregated costs of electronic discharge communication tool from project estimate (\$C 2016)

\begin{tabular}{|c|c|c|}
\hline & & $\begin{array}{l}\text { Estimated } \\
\text { cost (\$C) }\end{array}$ \\
\hline \multirow[t]{7}{*}{ Development } & Project charter and scope & 3000 \\
\hline & $\begin{array}{l}\text { Focus groups (including } 12 \\
\text { sessions and reports) }\end{array}$ & 18000 \\
\hline & Data and security model & 6250 \\
\hline & $\begin{array}{l}\text { Initial prototype (including interface } \\
\text { workflow, user management and } \\
\text { systems interface) }\end{array}$ & 31250 \\
\hline & $\begin{array}{l}\text { Joint application (including working } \\
\text { session and development) }\end{array}$ & 15000 \\
\hline & Beta testing & 10000 \\
\hline & Application fine tuning & 6250 \\
\hline \multirow[t]{2}{*}{ Implementation } & $\begin{array}{l}\text { Transfer to test environment } \\
\text { (including application installation, } \\
\text { interface configuration, workflows } \\
\text { and configuration) }\end{array}$ & 6250 \\
\hline & $\begin{array}{l}\text { Performance testing and } \\
\text { modifications (including interface, } \\
\text { workflow, load testing and security) }\end{array}$ & 12500 \\
\hline \multirow{2}{*}{$\begin{array}{l}\text { Training and } \\
\text { help }\end{array}$} & Training & 3000 \\
\hline & $\begin{array}{l}\text { Documentation and online help } \\
\text { (including administration manuals, } \\
\text { end user guide and patient guide) }\end{array}$ & 5000 \\
\hline Maintenance & Ongoing modifications and support & 5000 \\
\hline Tax & Goods and services tax (5\%) & 6075 \\
\hline \multicolumn{2}{|l|}{ Total cost } & 127575 \\
\hline \multicolumn{2}{|c|}{ Average cost per patient } & 13.33 \\
\hline
\end{tabular}

determined to be $\$$ C22.28. Average dictation time from 10 physicians was $10.87 \mathrm{~min}$ (range: $4-22 \mathrm{~min}$ ), and the average cost per minute of transcription was \$C2.05.

\section{Cost of eDCT}

From the project estimate, the estimated incremental cost of eDCT (on the backdrop of a functioning hospital information system platform) was $\$ \mathrm{C} 127575$. This estimate broadly captured costs of tool development, tool implementation, training and assistance, maintenance and taxation. After a 3-year amortisation at a $1 \%$ annual interest rate, the monthly payment amount for the tool was \$C3598. This resulted in an average cost per patient to $\$ \mathrm{C} 13.33$ (table 1).

\section{Patient population}

Between January 2012 and December 2013, 1953 patients were approached for study inclusion, of which 1399 MTU patients were randomised to discharge via the eDCT $(n=701)$ or traditional dictation methods $(n=698)$. Balance was achieved between age, sex and comorbidities between treatment and control. A full description of the patient population has been reported elsewhere. ${ }^{1}$ Fortyfive patients were missing index hospitalisation costs and were excluded from the cost analysis. An additional 
Table 2 Clinical effectiveness of eDCT by trial arm

\begin{tabular}{|c|c|c|c|c|c|c|}
\hline & \multicolumn{3}{|c|}{ eDCT } & \multicolumn{3}{|c|}{ Usual care } \\
\hline & $\mathbf{n}$ & Events & $95 \% \mathrm{Cl}$ & $\mathbf{n}$ & Events & $95 \% \mathrm{Cl}$ \\
\hline Total mortality at 3 months (\%) & 701 & $39(5.6)$ & 27.1 to 50.9 & 698 & $52(7.5)$ & 38.4 to 65.6 \\
\hline Average baseline utility & 564 & 0.294 & 0.267 to 0.321 & 566 & 0.309 & 0.283 to 0.336 \\
\hline
\end{tabular}

eDCT, electronic discharge communication tool.

28 patients in the treatment arm and 33 patients in the control arm did not complete the HUI-3, thus, utility scores were not available for these patients.

\section{Clinical effectiveness}

Death, readmissions and health-related quality of life were captured and calculated to measure clinical effectiveness (health benefit) of the eDCT (table 1). Specifically, $5.56 \%$ of patients in the eDCT arm died within 90 days of discharge compared with $7.45 \%$ in the usual care arm. The average number of readmissions was 0.46 for the eDCT group and 0.41 for the usual care group. Utilities at baseline and 90 days were similar between both arms (table 2).

For the index hospitalisation, the average cost per patient was higher in the usual care arm (table 3; \$C17506.71 compared with \$C16 832.36). The average number of readmissions was higher in the eDCT arm compared with usual care (0.46 vs 0.41$)$. Both trial arms had the same average number of ED visits (0.78); however, the average cost for ED visits was higher in the eDCT group (\$C757.83 compared with \$C683.01). The eDCT arm had a lower average number of ambulatory care visits (5.23 compared with 5.69) and physician claims (8.04 compared with 8.68). Finally, in the eDCT arm, the total average cost per patient (including all costs) was \$C32892 compared with \$C32059 in the usual care arm.

\section{Incremental cost-effectiveness ratio}

In the base case, the cost per QALY gained was \$C239933 in the eDCT arm compared with usual care. The cost per death avoided was $\$ \mathrm{C} 44051$, and the cost per readmission gained was \$C17490 in the eDCT arm compared with usual care.

\section{Sensitivity and scenario analyses}

When increasing the cost of the eDCT by $25 \%$, the cost per QALY gained increased to \$C240 893, the cost per death avoided increased to \$C44 227 and the cost per readmission gained increased to $\$ \mathrm{C} 17560$. When the costs of eDCT were decreased by $25 \%$, the cost per QALY gained decreased to $\$$ C238 974, the cost per death avoided decreased to $\$ \mathrm{C} 43875$ and the cost per readmission gained decreased to $\$ \mathrm{C} 17421$.
In the first scenario analysis (internal transcription using non-speech recognition system), it was assumed that 7418 discharge summaries could be transcribed per month with an average cost per patient of $\$ C 45.75$. The ICERs did not differ from the base case results (cost per QALY gained: \$C233 170, cost per death avoided: \$C42 809 and cost per readmission gained: \$C16 997). In the second scenario analysis (internal transcription using speech recognition system), it was assumed that 8240 discharge summaries could be transcribed per month with an average cost per patient of \$C40.55. This scenario also did not impact the estimated ICERs.

\section{Uncertainty: bootstrap analysis}

There is significant uncertainty in results with similar percentages of the samples falling into each of the cost-effective quadrants (figure 1). A total of $29.2 \%$ of the samples resulted in eDCT being both more effective and more costly, $29.2 \%$ of the samples resulted in eDCT being less costly and more effective, $23.9 \%$ of the samples resulted in eDCT being less effective and more costly and finally, $17.7 \%$ of the samples resulted in eDCT being less costly and less effective.

\section{DISCUSSION}

This prospective economic evaluation of an eDCT completed as part of an RCT found that eDCT produces cost savings relating to the immediate costs associated with producing discharge summaries at the time of discharge (\$C13.33 compared with \$C22.28). The cost per QALY gained with eDCT compared with usual care was \$C239 933, the cost per death avoided was \$C44051 and the cost per readmission gained was $\$ C 17490$. The large ICERs are due to the approximately \$C800 difference between arms with minimal effect on clinical outcomes. Moreover, when uncertainty is assessed, this evaluation resulted in relative equivalence for both the costs and the outcomes with approximately equal simulations resulting in higher costs and effects as lower costs and effects.

Given the neutral clinical effectiveness results, a cost-minimisation analysis may be considered. Within this framework, only the differences in costs are considered, 
Table 3 Healthcare resource utilisation by trial arm (\$C 2016)

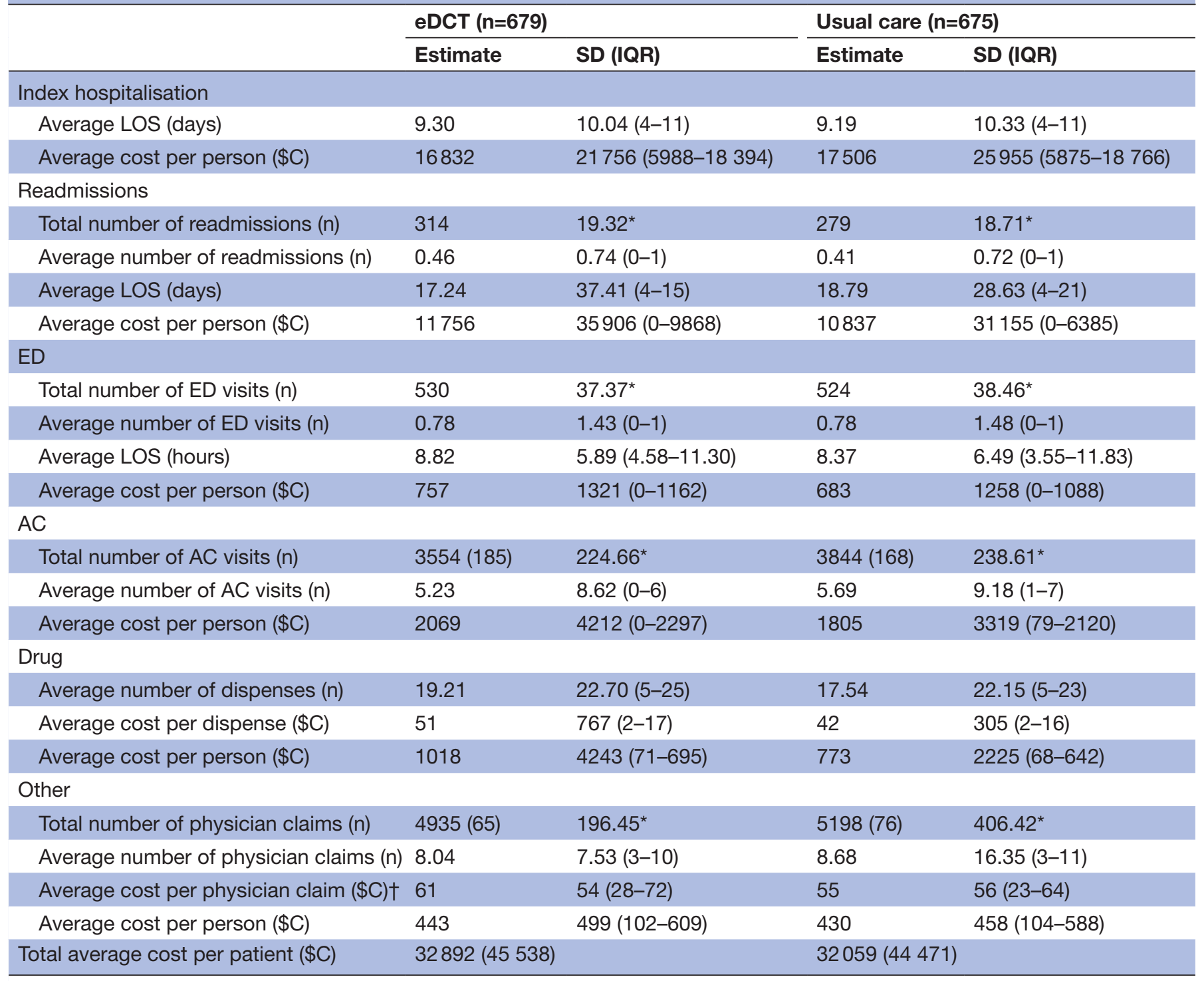

*SE.

†Includes imputed cost for patients missing physician claims data $(n=58)$.

AC, ambulatory care; ED, emergency department; eDCT, electronic discharge communication tool; LOS, length of stay.

as the outcomes are considered equivalent, with the least expensive option identified as the most attractive choice (achievement of the same clinical outcome for lower cost).${ }^{14}$ Our findings in the context of cost minimisation

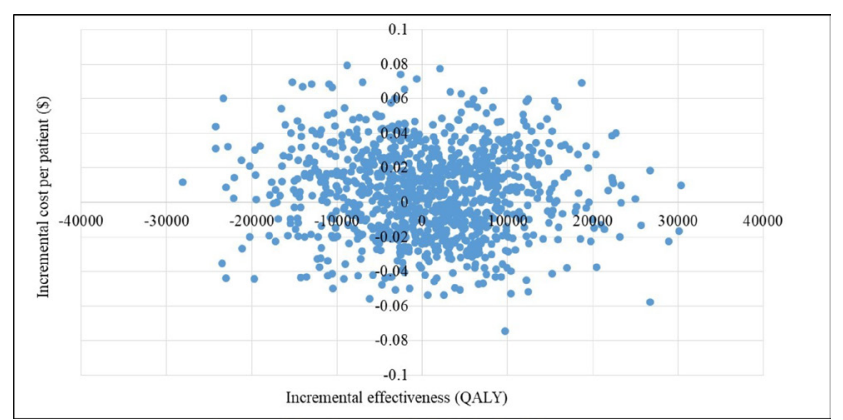

Figure 1 Uncertainty bootstrap analysis. QALY, qualityadjusted life year. are that eDCT is about \$C9 less expensive per patient than usual care. Based on this result, the use of an eDCT may be cost saving. Importantly, the cost of the eDCT used in this analysis only considered the costs of the pilot project estimate. It is expected that costs of largescale implementation may differ from the costs reported in this analysis. Other costs to consider include: the acquisition of the eDCT by the healthcare system, the introduction of the eDCT into existing infrastructure (including current electronic medical records), ensuring appropriate infrastructure and systems for community care physicians and ongoing software and network maintenance. However, establishing an eDCT is a relatively fixed cost; as the number of patients discharged through the eDCT increases, the per patient cost decreases. In contrast, traditional discharge is a variable cost whereby the total cost increases with each patient. Due to this, it 
is quite likely that the per patient cost associated with the eDCT would decrease further in the context of a largescale adoption.

Another consideration for the adoption of eDCTs is the maturity of the existing electronic management platforms within a healthcare system. For instance, in systems that have highly developed eHealth platforms, the adoption of an eDCT may be more economically attractive. The development of an additional page/tool and its incorporation into a well-managed and comprehensive system would be expected to be low cost, highly feasible and easily integrated into care delivery. Under these conditions, costs of electronic discharge may be expected to be lower than usual care. In contrast, systems with non-existent or minimally developed eHealth platforms will require major investment to implement an eDCT. For these systems, remaining with usual care or saving for the next technological advancement may be reasonable. In this scenario, eDCT is expected to be more expensive than usual care and without significant clinical benefit. It is also in this situation where adoption of an EDCT would require major trade-offs with other health technologies that may improve clinical outcomes.

In this trial, eDCT did not demonstrate an improvement in clinical outcomes. This is an important finding when considering the opportunity cost associated with an adoption decision. Specifically, under a limited budget, the adoption of these tools may require trading off with other life-saving or clinically beneficial tools. However, the outcomes assessed (death and readmissions) may be perceived as too narrow a lens to assess effectiveness when making adoption decisions. For example, many systems have adopted the quadruple aim to ground their perspective on healthcare system goals and performance. ${ }^{2}$ This model for optimising health system performance suggests considering clinical benefit, cost-effectiveness, patient experience and healthcare provider satisfaction. These four points could also be considered when facing adoption and implementation considerations. Specifically, patients in the eDCT arm were easily provided with a printout of their discharge summary as they were leaving the hospital. Through this mechanism, patients are also able to share information with other care team members and take a more active role in their healthcare; two important components of high-quality healthcare. ${ }^{15}$ In addition, in a cohort study of acute and community care physicians completed alongside this RCT, acute care physicians preferred eDCT over traditional dictation and discharge methods. ${ }^{16}$ Acknowledging this user preference may further increase provider satisfaction. Thus, when a framework such as the quadruple aim is adopted, eDCT may be viewed as an important step towards healthcare system improvement in 3 of the 4 domains (cost, patient satisfaction and provide satisfaction).

Our study had limitations. First, the costs of electronic discharge and traditional dictation are expected to vary across organisations and healthcare systems. Moreover, estimates for the cost of discharge mechanism are context specific and will depend on the number of patients discharged, the existing infrastructure and the eDCT itself. Organisations facing adoption decisions should consider their own costing and resources to inform policy decisions. Second, a small number of patients $(n=45)$ were lost to follow-up resulting in missing data; however, these patients were removed from the analysis and were similar to the patients who were not lost to follow-up. Third, the RCT permitted physician cross-over between trial arms. More specifically, due to the unit of randomisation being the patient, a physician may have used the eDCT to discharge one patient and then immediately switched to usual care to discharge the following patient. This concurrent exposure to the eDCT, which helped ensure balanced patient groups in the RCT, may have also improved the quality of the discharge summary in the usual care arm. Thus, the true benefit of eDCT may have been underestimated, which would in turn produce an overestimate of the cost per QALY. Finally, this study was restricted to the outcomes captured by the RCT. Specifically, differences in avoidable and unavoidable deaths were not divided in the RCT and are therefore not reflected in this analysis.

\section{CONCLUSIONS}

This prospective economic evaluation of a novel eDCT found that there were no differences in the costs and clinical benefits compared with usual care. However, there were immediate cost savings in transcription costs when eDCT is adopted that, when considered alongside positive patient and provider experiences with the eDCT, increase the impetus for healthcare organisations to invest in such systems. However, when considering the adoption of eDCTs, healthcare systems should critically assess their current eHealth infrastructure and the required investment to adopt eDCTs.

Acknowledgements We gratefully acknowledge the other investigators who contributed to the design of the RCT: Jayna Holroyd-Leduc, Danielle Southern, Ward Flemons, Maeve O'Beirne, Michael Hill, Alan Forster and Deborah E White.

Contributors LKS, M-JS, WAG and FC contributed to the conceptualisation of the study. M-JS and WAG led all aspects of the primary clinical analysis of the randomised controlled trial (RCT). FC provided oversight and supervision of the economic analysis of the RCT. LKS completed the analysis with supervision from FC, M-JS and WAG. LKS drafted the manuscript with FC, M-JS and WAG providing critical revisions. All authors approved the final version.

Funding This RCT was funded by the Canadian Institutes of Health Research (CIHR) and was further supported by the Medical Teaching Unit (supporting implementation of eDCT) Alberta Health Services (adoption and implementation) and W21C (development and research).

Competing interests None declared.

Patient consent Obtained.

Ethics approval Conjoint Health Research Ethics Board at the University of Calgary (no 23469).

Provenance and peer review Not commissioned; externally peer reviewed.

Data sharing statement Any unpublished aggregate data will be made available upon request by the corresponding author.

Open Access This is an Open Access article distributed in accordance with the Creative Commons Attribution Non Commercial (CC BY-NC 4.0) license, which 
permits others to distribute, remix, adapt, build upon this work non-commercially, and license their derivative works on different terms, provided the original work is properly cited and the use is non-commercial. See: http://creativecommons.org/ licenses/by-nc/4.0/

(c) Article author(s) (or their employer(s) unless otherwise stated in the text of the article) 2017. All rights reserved. No commercial use is permitted unless otherwise expressly granted.

\section{REFERENCES}

1. Santana MJ, Holroyd-Leduc J, Southern DA, et al. A randomised controlled trial assessing the efficacy of an electronic discharge communication tool for preventing death or hospital readmission. BMJ Qual Saf 2017;26:993-1003.

2. Bodenheimer T, Sinsky C. From triple to quadruple aim: care of the patient requires care of the provider. Ann Fam Med 2014;12:573-6.

3. Chin JP, Diehl VA, Norman KL. Development of an instrument measuring user satisfaction of the human-computer interface. $\mathrm{CHI}$ '88 Proceedings of SIGCHI Conference on Human Factors in Computing Systems. New York: ACM Press, 1988.

4. Okoniewska BM, Santana MJ, Holroyd-Leduc J, et al. The seamless transfer-of-care protocol: a randomized controlled trial assessing the efficacy of an electronic transfer-of-care communication tool. BMC Health Serv Res 2012;12:414.

5. Santana MJ, Holroyd-Leduc J, Flemons WW, et al. The seamless transfer of care: a pilot study assessing the usability of an electronic transfer of care communication tool. Am J Med Qual 2014;29:476-83.

6. Sevick LK, Esmail R, Tang K, et al. A systematic review of the cost and cost-effectiveness of electronic discharge communications. BMJ Open 2017;7:e014722.
7. Niedbala RS, Kardos KW, Fritch DF, et al. Passive cannabis smoke exposure and oral fluid testing. II. Two studies of extreme cannabis smoke exposure in a motor vehicle. J Anal Toxicol 2005;29:607-15.

8. Kopach R, Sadat S, Gallaway ID, et al. Cost-effectiveness analysis of medical documentation alternatives. Int $J$ Technol Assess Health Care 2005;21:126-31.

9. Moore C, Coulter C, Uges D, et al. Cannabinoids in oral fluid following passive exposure to marijuana smoke. Forensic Sci Int 2011;212:227-30.

10. Furlong W, Feeny D, Torrance G, et al. Multiplicative multi-attribute utility function for the health utilities index mark 3 (hui3) system: a technical report: McMaster University Centre for Health Economics and Policy Analysis. Working Paper No: 98-11 December 1998.

11. Feeny D, Furlong W, Boyle M, et al. Multi-attribute health status classification systems. Health Utilities Index. Pharmacoeconomics 1995; 7:490-502.

12. Feeny D, Torrance GW, Furlong WJ. Chapter 26: "Health Utilities Index". Spilker B, ed. Quality of life and pharmacoeconomics in clinical trials. 2nd edn. Philadelphia: Lippincott-Raven Press, 1996:239-52.

13. Canadian institue for health information. Comprehensive Ambulatory Classification System (CACS). CIHI; 1996-2017 https:// www.cihi.ca/en/comprehensive-ambulatory-classification-systemcacs.

14. Drummond M, Sculpher M, Torrance G, et al. Methods for the economic evaluation of health care programmes. 3rd edn. Oxford, UK: Oxford University Press, 2005.

15. Rabi $D$, Chemali $B$. Health information exchange: engaging providers in health care innovation: Towards "Person-Centred" Integrated Health in Alberta, 2017 Edmonton. Alberta.

16. de Grood C, Eso K, Santana MJ. Physicians' experience adopting the electronic transfer of care communication tool: barriers and opportunities. J Multidiscip Healthc 2015;8:21-31. 